\title{
Robust Low-profile Metasurface-Enabled Wearable Antennas for Off-Body Communications
}

\author{
Zhi Hao Jiang and Douglas H. Werner \\ Department of Electrical Engineering \\ The Pennsylvania State University \\ University Park, PA, USA \\ dhw@psu.edu
}

\begin{abstract}
This paper presents a low-profile metasurfaceenabled wearable antenna operating in the newly released medical wireless body-are network (MBAN) band from 2.36 to 2.40 GHz for off-body communications. The performance of the antenna was made possible by the introduction of a properly designed anisotropic metasurface which consists of only a 2 by 2 array of I-shaped resonating elements. Together with the finitesized metallic ground plane on the back of the metasurface, it provides a $+90^{\circ}$ reflection phase band to achieve the desired impedance matching for the planar monopole feed above the metasurface. The integrated antenna was fabricated and characterized, showing a strong correspondence between simulated and measured results. The resulting antenna has a remarkably low profile of only one-fiftieth of the operating wavelength, all while achieving a $5.5 \%-10 \mathrm{~dB}$ impedance bandwidth from 2.30 to $2.43 \mathrm{GHz}$, a gain of around $6.2 \mathrm{dBi}$, and a font-to-back ratio higher than $23 \mathrm{~dB}$, thus making it a promising candidate for incorporation into various wearable systems.
\end{abstract}

Index Terms-anisotropic metasurface, medical body-area network, wearable antennas.

\section{INTRODUCTION}

The field of wearable computing systems and sensing devices has experienced rapid development over the past decade due to their great potential for applications in numerous diverse areas such as health monitoring, patient tracking, battlefield survival, and so on [1]. As one of the key components in the system, wearable antennas have received much attention in both academia and industry due to their unconventional operating environment - a region that is in extremely close proximity to the human body. As a result of such a fact, the loading of lossy human tissue makes the design of a high radiation efficiency antenna challenging when it is also desirable for it to possess light-weight and low-profile characteristics. Simultaneously, the impact of a wearable antenna on human tissue, characterized by the specific absorption rate (SAR), also needs to be minimized.

Thus far, several types of designs have been proposed and investigated for their suitability as wearable antennas, such as vertical monopoles, planar microstrip monopoles [2], planar inverted-F antennas [3], microstrip patch antennas [4], and cavity-backed or planar waveguide fed slot antennas $[5,6]$. These antennas, however, possess either very narrow bandwidth, large footprint, and/or high profile, making them of only limited use in wearable systems. More recently, artificial magnetic conducting (AMC) surfaces have been proposed and demonstrated to enable low profile wearable antennas with a high degree of isolation from the human body [7, 8]. But, some drawbacks still exist in the current isotropic AMC backed antenna designs such as large footprint [7] or poor front-toback ratio [8].
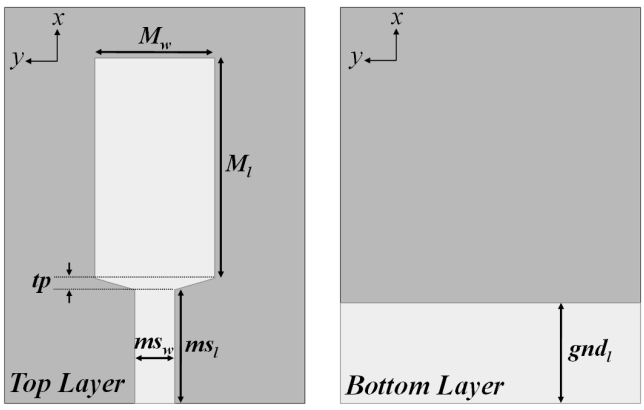

(a)
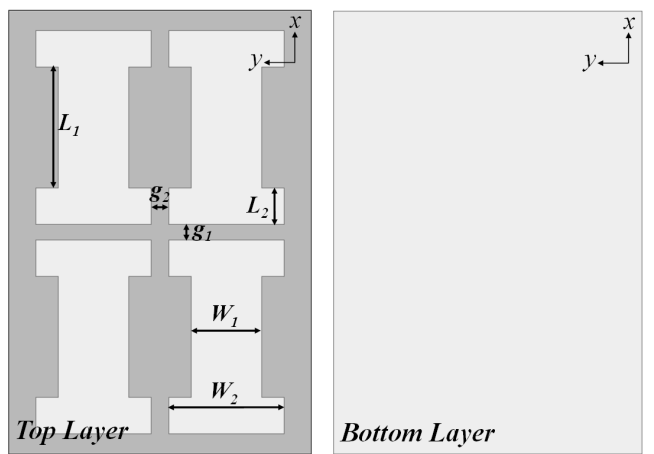

(b)

Fig. 1. (a) Top and bottom views of the top monopole layer. (b) Top and bottom views of the finite sized metasurface layer with a metallic backing. The optimized dimensions are $G_{x}=62 \mathrm{~mm}, G_{y}=42 \mathrm{~mm}, A_{x}=$ $39 \mathrm{~mm}, A_{y}=30 \mathrm{~mm}, d_{1}=1.5 \mathrm{~mm}, M_{w}=12 \mathrm{~mm}, M_{l}=21.8 \mathrm{~mm}, \mathrm{tp}=$ $1.15 \mathrm{~mm}, \mathrm{~ms}_{w}=4 \mathrm{~mm}, \mathrm{~ms}_{l}=11.3 \mathrm{~mm}$, gnd $_{l}=10 \mathrm{~mm}, L_{1}=16.86 \mathrm{~mm}, L_{2}=$ $5.1 \mathrm{~mm}, W_{1}=9.75 \mathrm{~mm}, W_{2}=16 \mathrm{~mm}, g_{l}=2.15 \mathrm{~mm}$, and $g_{2}=2.49 \mathrm{~mm}$. The substrate material is Rogers RO3003 $\left(\varepsilon_{r}=3, \delta_{\text {tan }}=0.0013\right)$. 
In this paper, we propose a conformal antenna with a compact footprint and a low-profile for future wearable devices functioning in the new $2.36-2.4 \mathrm{GHz}$ MBAN band recently released by the Federal Communication Commission (FCC) [9] due to its clean spectrum and low interference sources. First, the antenna configuration and design approach is discussed in Section II, along with simulated antenna performance. The experimental results are presented in Section III.
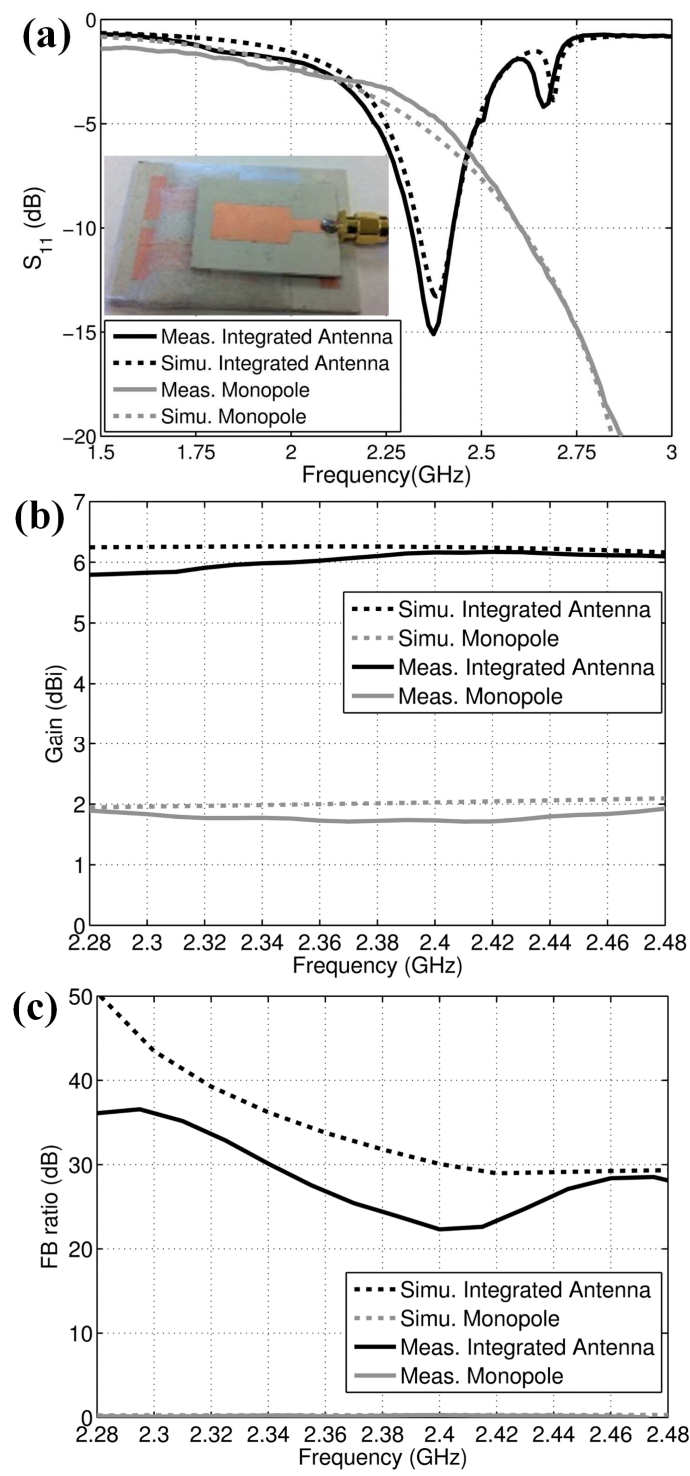

Fig. 2. Simulated and measured (a) $S_{11}$, (b) gain, and (c) FB ratio of the monopole alone and the integrated antenna. The inset of (a) shows a photo of the fabricated antenna.

\section{ANTENNA DESIGN}

The planar metasurface-enabled conformal antenna is comprised of two components separated by a thin foam spacer - a planar monopole antenna on the top (see Fig. 1(a)) and on the bottom a finite sized anisotropic metasurface with a metallic sheet backing (see Fig. 1(b)). The top monopole and the bottom metasurface layers are $1.5 \mathrm{~mm}$ thick. The top planar monopole is fed by a microstrip while the metasurface layer is comprised of a two by two I-shaped array of unit cells. The Ishaped elements are oriented with their long axes in the $x$ direction, i.e. parallel to the monopole, for the purpose of providing efficient coupling.

During the first step of the design process, the geometrical dimensions of the ground plane backed I-shaped elements were tuned to yield a $+90^{\circ}$ reflection phase at around $2.4 \mathrm{GHz}$. The dimensions of the top monopole and the thickness of the foam spacer were then adjusted to achieve the optimal impedance match, high gain and high FB ratio at the targeted band. The final antenna design has a compact form factor of $0.5 \lambda_{0} \times 0.3 \lambda_{0} \times 0.028 \lambda_{0}$. It should be noted that the inductive reflection property of the metasurface is critical for accomplishing a matched impedance for the top monopole at a frequency range below its fundamental quarter-wavelength resonating mode. This is because within such a band, the input impedance of the monopole alone is capacitive and, hence, when loaded by the inductive impedance surface which stores more magnetic energy, the reactance of the integrated antenna can be suppressed, thus achieving a good impedance match.

The simulated results of both the monopole and the integrated antenna in free space are shown in Fig. 2. At the MBAN band, the monopole has a poor impedance match showing a $S_{11}$ higher than $-6 \mathrm{~dB}$. The integrated antenna, in contrast, exhibits a good impedance match with its $S_{I I}$ lower than $-10 \mathrm{~dB}$ from 2.32 to $2.43 \mathrm{GHz}$, i.e. around $4.7 \%$ bandwidth, which is wide enough to cover the MBAN band. The monopole alone has a simulated gain of around $2 \mathrm{dBi}$ whereas the integrated antenna has a simulated gain of around $6.2 \mathrm{dBi}$, as shown in Fig. 2(b). In terms of FB ratio, while the monopole has a near-zero value, the integrated metasurfaceenabled antenna has a simulated FB ratio greater than $29 \mathrm{~dB}$. Such a high FB ratio indicates that very little energy would be radiated into the tissue when such an antenna is placed on the human body. This property helps to minimize the SAR value and makes the antenna more robust to the loading effects of the human body, both of which are desirable characteristics for wearable applications.

\section{MEASUREMENTS}

The metasurface-enabled antenna was fabricated using standard PCB board etching and measured using a network analyzer and an anechoic chamber. As shown in Fig. 2, good agreement can be found between simulated and measured results. The measured $S_{11}$ of the integrated antenna has a $5.5 \%$ $-10 \mathrm{~dB}$ bandwidth ranging from 2.30 to $2.43 \mathrm{GHz}$, which is slightly broader than the simulation prediction due to a very small reduction in the quality factor. The measured gain values for both the monopole and the integrated metasurface-enabled 
antenna are slightly lower than the simulated results, but with a difference less than $0.4 \mathrm{~dB}$. The integrated antenna achieves a gain of $5.85 \mathrm{dBi}$ in the range from 2.30 to $2.43 \mathrm{GHz}$ within which the $S_{11}$ is below $-10 \mathrm{~dB}$. The measured FB ratio exhibits a value greater than $23 \mathrm{~dB}$. Further measurements reveal that the antenna performance is well maintained even at close proximity to the human body (not shown here).

\section{SPECIFIC ABSORPTION RATE (SAR) EVALUATION}

In order to evaluate the impact of the antenna on the human body, the SAR value, which represents a standard measure often used to estimate the absorption of electromagnetic power in human tissue, was further investigated using full-wave simulations. According to the specification provided by the FCC, the SAR values must be no greater than $1.6 \mathrm{~W} / \mathrm{kg}$ averaged over $1 \mathrm{~g}$ of tissue [10]. In our study, a low power input of only $10 \mathrm{~mW}$ was chosen to evaluate and compare the SAR performance of the planar monopole antenna and the proposed integrated antenna. The configurations are shown in Fig. 3(a) and Fig. 3(b) where the antennas are placed to a multilayer human tissue model. The skin, fat, mussel, and bone layers have thickness values of 2 $\mathrm{mm}, 5 \mathrm{~mm}, 20 \mathrm{~mm}$, and $13 \mathrm{~mm}$, respectively. The relative

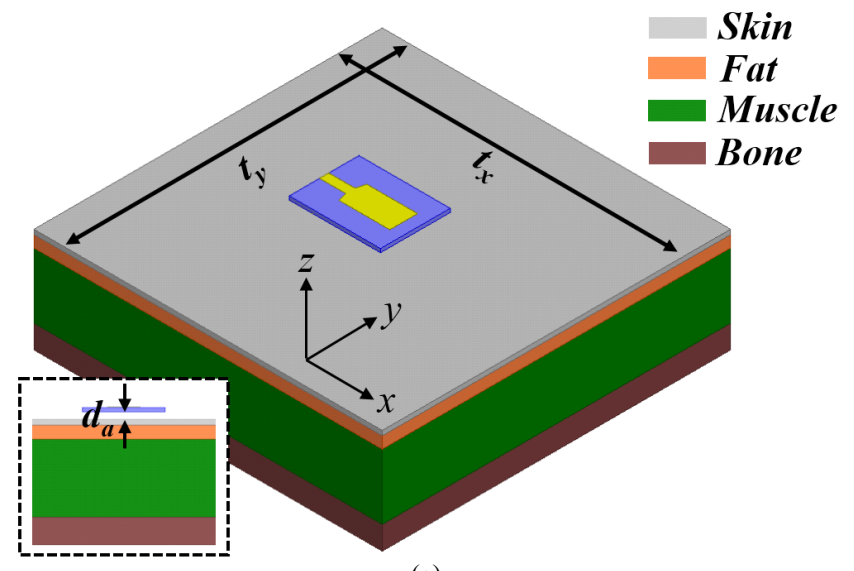

(a)

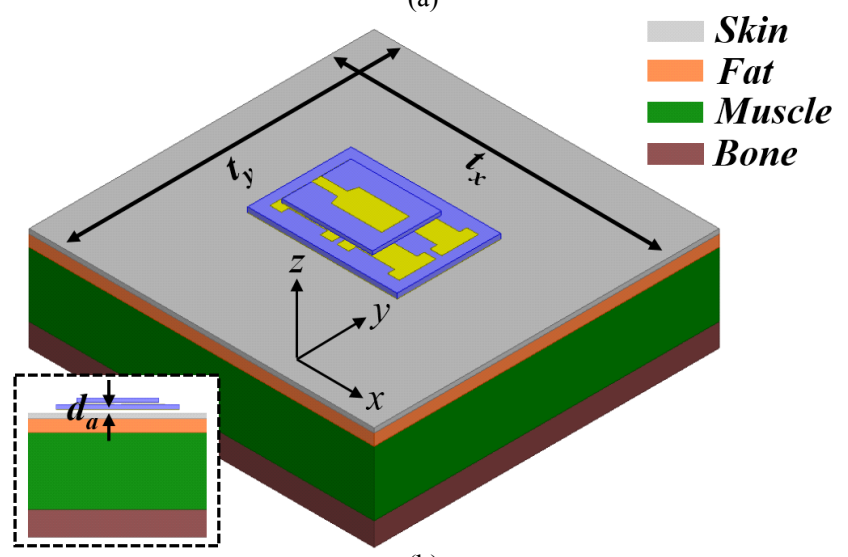

(b)

Fig. 5. Configurations of (a) the planar monopole antenna and (b) the integrated metasurface-enabled antenna when placed on a multilayer tissue model. The dimensions are $t_{x}=150 \mathrm{~mm}$ and $t_{y}=150 \mathrm{~mm}$.

permittivity values for the skin, fat, mussel, and bone layers are $37.95,5.27,52.67$, and 18.49 , respectively, while the conductivity values for the four layers are $1.49,0.11,1.77$, and $0.82 \mathrm{~S} / \mathrm{m}$. The material and dimension values for each layer were taken from previously reported studies [11].

The simulated averaged SAR is shown in Fig. 4(a) and Fig. 4(b). For the considered input power, the planar monopole antenna generates a peak SAR value of about 4.01 $\mathrm{W} / \mathrm{kg}$ due to its omnidirectional radiation characteristic.

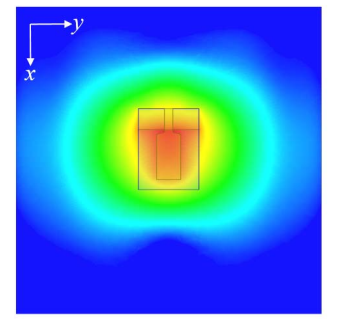

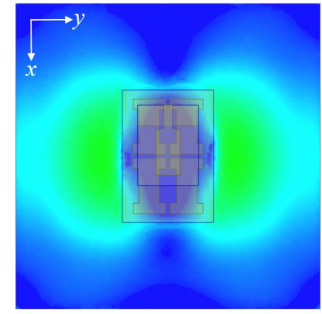

Average SAR $(\mathrm{W} / \mathrm{kg})$ $d_{a}=1 \mathrm{~mm}$

(a)

(b)

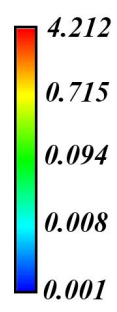

$2.38 \mathrm{GHz}$ $P_{i n}=10 \mathrm{~mW}$

Fig. 3. Simulated averaged SAR values for (a) the planar monopole and (b) the integrated metasurface-enabled antenna at $1 \mathrm{~mm}$ away from the multilayer tissue model.

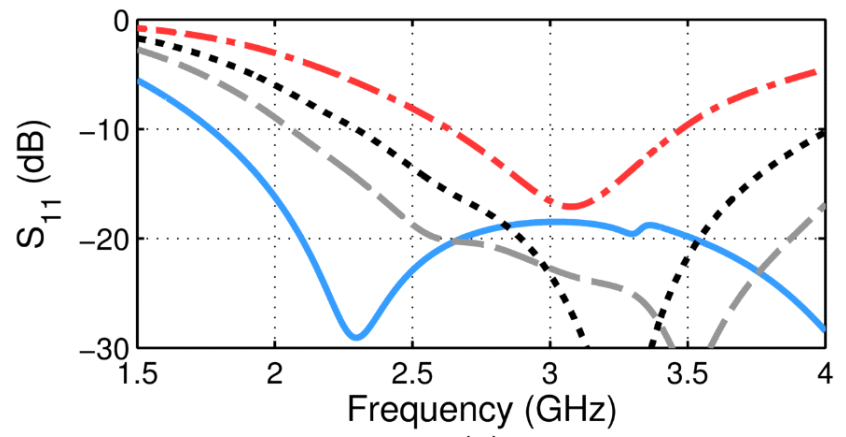

(a)

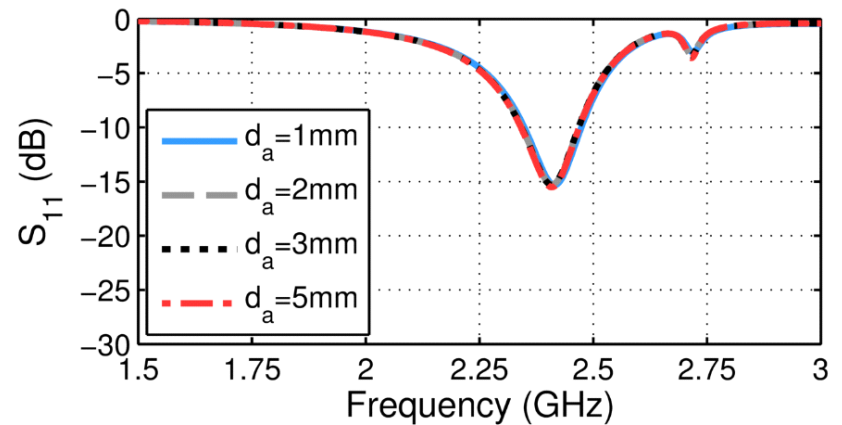

(b)

Fig. 4. Simulated $S_{11}$ for (a) the planar monopole and (b) the integrated metasurface-enabled antenna at different distances $\left(d_{a}\right)$ away from the multilayer tissue model.

Additional simulations show that even at a distance of $5 \mathrm{~mm}$ away from the multilayer tissue model, the monopole experiences a peak SAR value as high as $2.05 \mathrm{~W} / \mathrm{kg}$ with such a low input power of $10 \mathrm{~mW}$. With the metallic sheet backed metasurface included, however, a significant reduction is 
observed in the peak SAR value - dropping to $0.067 \mathrm{~W} / \mathrm{kg}$ even when the integrated metasurface-enabled antenna is only $1 \mathrm{~mm}$ away from the tissue. As displayed in Fig. 5(a) and Fig. 5(b), when being placed on the multilayer tissue model, the integrated metasurface-enabled antenna maintains a robust and independent input impedance with its $S_{11}<-10 \mathrm{~dB}$ at the targeted MBAN band. The $S_{I I}$ of the planar monopole shows a strong variation as a function of the distance between the antenna and the tissue model. In addition, even though its $S_{11}$ is below $-10 \mathrm{~dB}$ in a fairly broad frequency range, its gain varies in the range from -8 to $-4 \mathrm{dBi}$ (not shown here), indicating an ultralow radiation efficiency due to the absorption caused by human body loading. In contrast, the gain of the integrated antenna is well-maintained when it is placed on the human tissue model, showing a stable $6 \mathrm{dBi}$ gain at broadside (not presented here). This also explains the significantly higher SAR value observed in the planar monopole antenna case compared to integrated antenna case. In all, together with the fact that the antenna properties are robust to human body loading, the $98.7 \%$ reduction in SAR further manifests the superiority of the proposed integrated antenna, which ensures a highly efficient radiator that is isolated from the body even when placed in close proximity.

\section{CONCLUSIONS}

In conclusion, we have proposed and demonstrated a conformal antenna with a compact footprint, light-weight, and low-profile design for wearable applications. A finite sized metallic sheet backed anisotropic metasurface was employed to provide inductive loading, which enables impedance matching to the capacitive feed element in the MBAN band while simultaneously delivering the necessary isolation between the antenna and human tissue. The realized antenna accomplishes a $5.5 \%-10 \mathrm{~dB}$ bandwidth, a gain of around 6.2 $\mathrm{dBi}$, and a $\mathrm{FB}$ ratio greater than $23 \mathrm{~dB}$. The SAR simulations further prove the superiority of the proposed antenna, showing a low SAR value and a robust performance to human body loading.

\section{ACKNOWLEDGMENT}

This work was supported by the National Science Foundation ASSIST Nanosystems ERC under Award Number EEC-1160483. This work was also supported by the EMERALD Project, which is funded by the Provincia Autonoma di Trento under the "Bando Unità Ricerca 2011"

\section{REFERENCES}

[1] P. S. Hall and Y. Hao, Antenna and Propagation for Body-Centric Wireless Cummunications. Artech House, Norwood, MA, United States, 2012.

[2] M. N. Suma et al., "A wideband printed monopole antenna for 2.45 GHz WLAN applications," Microw. Opt. Technol. Lett., vol. 48, no. 5, pp. 871-873, May 2006

[3] P. J. Soh et al. , "Design of a broadband all-textile slotted PIFA," IEEE Trans. Antennas Propagat., vol. 60, no. 1, pp. 379-384, Jan. 2012.

[4] A. Alomainy et al., "Statistical analysis and performance evaluation for on-body radio propagation with microstrip patch antennas," IEEE Trans. Antennas Propagat., vol. 55, no. 1, pp. 245-248, Jan. 2007.

[5] N. Haga et al., "Characteristics of cavity slot antenna for body-area networks," IEEE Trans. Antennas Propagat., vol. 57, no. 4, pp. 837 843, Apr. 2009

[6] R. Moro et al., "Wearable textile antenna in substrate integrated waveguide technology," Electron. Lett., vol. 48, no. 16, pp. 985-986, Aug. 2012.

[7] S. Zhu et al., "Dual-band wearable textile antenna on an EBG substrate," IEEE Trans. Antennas Propagat., vol. 57, no. 4, pp. 926935, Apr. 2009.

[8] H. R. Raad et al., "Flexible and compact AMC based antenna for telemedicine applications," IEEE Trans. Antennas Propagat., vol. 61, no. 2, pp. 524-531, Feb. 2013.

[9] Low-Rate Wireless Personal Area Networks (LR-WPANs) Amendment 4, IEEE Standard 802.15.4j, 2013.

[10] http://www.fcc.gov/encyclopedia/specific-absorption-rate-sar-cellulartelephones

[11] M. A. Stuchly and S. S. Stuchly, "Dielectric properties of biological substances-tabulated," J. Microw. Power, vol. 15, no. 1, pp. 19-26, Jan. 1980. 